\title{
CLASSIFYING ORBITS OF THE AFFINE GROUP OVER THE INTEGERS
}

\author{
LEONARDO MANUEL CABRER AND DANIELE MUNDICI
}

\begin{abstract}
For each $n=1,2, \ldots$, let $\mathrm{GL}(n, \mathbb{Z}) \ltimes \mathbb{Z}^{n}$ be the affine group over the integers. For every point $x=\left(x_{1}, \ldots, x_{n}\right) \in \mathbb{R}^{n}$ let $\operatorname{orb}(x)=\left\{\gamma(x) \in \mathbb{R}^{n} \mid \gamma \in \mathrm{GL}(n, \mathbb{Z}) \ltimes \mathbb{Z}^{n}\right\}$. Let $G_{x}$ be the subgroup of the additive group $\mathbb{R}$ generated by $x_{1}, \ldots, x_{n}, 1$. If $\operatorname{rank}\left(G_{x}\right) \neq n$ then $\operatorname{orb}(x)=\left\{y \in \mathbb{R}^{n} \mid G_{y}=G_{x}\right\}$. Thus, $G_{x}$ is a complete classifier of orb(x). By contrast, if $\operatorname{rank}\left(G_{x}\right)=n$, knowledge of $G_{x}$ alone is not sufficient in general to uniquely recover $\operatorname{orb}(x)$ : as a matter of fact, $G_{x}$ determines precisely $\max \left(1, \frac{\phi(d)}{2}\right)$ different orbits, where $d$ is the denominator of the smallest positive nonzero rational in $G_{x}$, and $\phi$ is Euler function. To get a complete classification, rational polyhedral geometry provides an integer $1 \leq c_{x} \leq \max (1, d / 2)$ such that $\operatorname{orb}(y)=\operatorname{orb}(x)$ iff $\left(G_{x}, c_{x}\right)=\left(G_{y}, c_{y}\right)$.
\end{abstract}

\section{INTRODUCTION}

Throughout we let $\mathrm{GL}(n, \mathbb{Z}) \ltimes \mathbb{Z}^{n}$ denote the group of affine transformations of the form $x \mapsto$ $\mathcal{U} x+t$ for $x \in \mathbb{R}^{n}$, where $t \in \mathbb{Z}^{n}$ and $\mathcal{U}$ is an integer $n \times n$ matrix with $\operatorname{det}(\mathcal{U})= \pm 1$. The dimension of the ambient space $\mathbb{R}^{n}$ will always be clear from the context. We let $\operatorname{orb}(x)$ denote the $\left(\mathrm{GL}(n, \mathbb{Z}) \ltimes \mathbb{Z}^{n}\right)$-orbit of $x \in \mathbb{R}^{n}$,

$$
\operatorname{orb}(x)=\left\{\gamma(x) \in \mathbb{R}^{n} \mid \gamma \in \mathrm{GL}(n, \mathbb{Z}) \ltimes \mathbb{Z}^{n}\right\} .
$$

In this self-contained paper, generalizing [7] we classify $\left(\mathrm{GL}(n, \mathbb{Z}) \ltimes \mathbb{Z}^{n}\right)$-orbits for all $n$ : every point in $\mathbb{R}^{n}$ is assigned an invariant, in such a way that two points have the same $\left(\mathrm{GL}(n, \mathbb{Z}) \ltimes \mathbb{Z}^{n}\right)$ orbit iff they have the same invariant. For each $x=\left(x_{1}, \ldots, x_{n}\right) \in \mathbb{R}^{n}$, the main invariant considered in this paper is the group

$$
G_{x}=\mathbb{Z}+\mathbb{Z} x_{1}+\mathbb{Z} x_{2}+\cdots+\mathbb{Z} x_{n}
$$

generated by $1, x_{1}, x_{2}, \ldots, x_{n}$ in the additive group $\mathbb{R}$. From Corollaries 16 and 17 it follows that $G_{x}$ completely classifies the $\left(\mathrm{GL}(n, \mathbb{Z}) \ltimes \mathbb{Z}^{n}\right)$-orbit of any $x \in \mathbb{R}^{n}$ when $\operatorname{rank}\left(G_{x}\right) \neq n$.

If $\operatorname{rank}\left(G_{x}\right)=n, G_{x}$ determines precisely $\max \left(1, \frac{\phi(d)}{2}\right)$ different $\left(\mathrm{GL}(n, \mathbb{Z}) \ltimes \mathbb{Z}^{n}\right)$-orbits, where $d$ is the denominator of the smallest positive nonzero rational in $G_{x}$ and $\phi$ is Euler function. To get a complete invariant for $\operatorname{orb}(x)$, rational polyhedral geometry equips $x$ with an integer $1 \leq c_{x} \leq \max (1, d / 2)$ which, together with $G_{x}$, completely classifies $\operatorname{orb}(x)$. Thus in Theorem 15 we prove: $\operatorname{orb}(x)=\operatorname{orb}(y)$ iff $\left(G_{x}, c_{x}\right)=\left(G_{y}, c_{y}\right)$. In case $\operatorname{rank}\left(G_{x}\right) \neq n, c_{x}=1$.

While the study of the orbits of the groups $\mathrm{GL}(2, \mathbb{Z}), \mathrm{SL}(2, \mathbb{Z}), \mathrm{SL}(2, \mathbb{Z})_{+}$requires techniques from various mathematical areas [1, 2, 4, 8, 9, 10, a main possible reason of interest in our classification stems from the pervasive and novel role played by rational polyhedral geometry 3 , through the fundamental notion of a (Farey) regular simplex [6]. Regular simplexes in $\mathbb{R}^{n}$ are the affine counterparts of regular cones in $\mathbb{Z}^{n+1}$. Regular fans are complexes of regular cones, and yield a combinatorial classification of nonsingular toric varieties [3].

\section{Preliminaries: AfFine SPACeS AND Regular SimpleXes}

A point $y=\left(y_{1}, \ldots, y_{n}\right) \in \mathbb{R}^{n}$ is said to be rational if each coordinate $y_{i}$ is a rational number.

Key words and phrases. Affine group over the integers, orbit, $\mathrm{GL}(n, \mathbb{Z}) \ltimes \mathbb{Z}^{n}$-orbit, complete invariant, rational simplex, Farey regular simplex.

This research was supported by a Marie Curie Intra European Fellowship within the 7th European Community Framework Program (ref. 299401-FP7-PEOPLE-2011-IEF).

2000 Mathematics Subject Classification. Primary: 37C85. Secondary: 11B57, 22F05, 37A45, 06F20, 46L80. 
A rational hyperplane $H \subseteq \mathbb{R}^{n}$ is a set of the form $H=\left\{z \in \mathbb{R}^{n} \mid\langle h, z\rangle=r\right\}$, for some nonzero vector $h \in \mathbb{Q}^{n}$ and $r \in \mathbb{Q}$. Here $\langle-,-\rangle$ denotes scalar product. A rational affine space $A$ in $\mathbb{R}^{n}$ is an intersection of rational hyperplanes in $\mathbb{R}^{n}$. For any $S \subseteq \mathbb{R}^{n}$ the affine hull aff $(S)$ is the set of all affine combinations in $\mathbb{R}^{n}$ of elements of $S$. Thus $z$ belongs to aff $(S)$ iff there are $w_{1}, \ldots, w_{k} \in S$ and $\lambda_{1}, \ldots, \lambda_{k} \in \mathbb{R}$ such that $\lambda_{1}+\cdots+\lambda_{k}=1$ and $z=\lambda_{1} \cdot w_{1}+\cdots+\lambda_{k} \cdot w_{k}$. A set $\left\{y_{1}, \ldots, y_{m}\right\}$ of points in $\mathbb{R}^{n}$ is said to be affinely independent if none of its elements is an affine combination of the remaining elements. One then easily sees that a subset $E$ of $\mathbb{R}^{n}$ is a rational affine subspace of $\mathbb{R}^{n}$ iff there exist $v_{0}, \ldots, v_{m} \in \mathbb{Q}^{n}$ such that $E=\operatorname{aff}\left(v_{0}, \ldots, v_{m}\right)$. For $0 \leq m \leq n$, an $m$-simplex in $\mathbb{R}^{n}$ is the convex hull $T=\operatorname{conv}\left(v_{0}, \ldots, v_{m}\right)$ of $m+1$ affinely independent points $v_{0}, \ldots, v_{m} \in \mathbb{R}^{n}$. The vertices $v_{0}, \ldots, v_{m}$ are uniquely determined by $T$. $T$ is said to be a rational simplex if its vertices are rational.

By the denominator $\operatorname{den}(y)$ of a rational point $y$ we understand the least common denominator of its coordinates. The homogeneous correspondent of a rational point $y=\left(y_{1}, \ldots, y_{n}\right) \in \mathbb{R}^{n}$ is the integer vector

$$
\widetilde{y}=\left(\operatorname{den}(y) \cdot y_{1}, \ldots, \operatorname{den}(y) \cdot y_{n}, \operatorname{den}(y)\right) \in \mathbb{Z}^{n+1} .
$$

Following [6], a rational $t$-simplex $T=\operatorname{conv}\left(v_{0}, \ldots, v_{t}\right) \subseteq \mathbb{R}^{n}$ is said to be (Farey) regular if the set $\left\{\tilde{v}_{0}, \ldots, \tilde{v}_{t}\right\}$ of homogeneous correspondents of the vertices of $T$ can be extended to a base of the free abelian group $\mathbb{Z}^{n+1}$. (Regular simplexes are called "unimodular" in [5].)

Lemma 1. For each rational point $x \in \mathbb{R}^{n}$ and $\psi \in \mathrm{GL}(n, \mathbb{Z}) \ltimes \mathbb{Z}^{n}$, $\operatorname{den}(x)=\operatorname{den}(\psi(x))$. Moreover, if $\operatorname{conv}\left(v_{0}, \ldots, v_{n}\right) \subseteq \mathbb{R}^{n}$ and $\operatorname{conv}\left(w_{0}, \ldots, w_{n}\right) \subseteq \mathbb{R}^{n}$ are regular $n$-simplexes, the following conditions are equivalent:

(i) $\operatorname{den}\left(v_{i}\right)=\operatorname{den}\left(w_{i}\right)$ for all $i \in\{0, \ldots, n\}$;

(ii) there exists $\gamma \in \mathrm{GL}(n, \mathbb{Z}) \ltimes \mathbb{Z}^{n}$ such that $\gamma\left(v_{i}\right)=w_{i}$ for each $i \in\{0, \ldots, n\}$.

Proof. It is easy to see that $\operatorname{den}(x)=\operatorname{den}(\psi(x))$, for every $\psi \in \mathrm{GL}(n, \mathbb{Z}) \ltimes \mathbb{Z}^{n}$. So (ii) $\Rightarrow$ (i) is immediately verified. For the converse direction (i) $\Rightarrow\left(\right.$ ii), the regularity of $\operatorname{conv}\left(v_{0}, \ldots, v_{n}\right)$ and $\operatorname{conv}\left(w_{0}, \ldots, w_{n}\right)$ yields bases $\left\{\tilde{v}_{0}, \ldots, \tilde{v}_{n}\right\}$ and $\left\{\tilde{w}_{0}, \ldots, \tilde{w}_{n}\right\}$ of the free abelian group $\mathbb{Z}^{n+1}$. There are integer matrices $\mathcal{M}$ and $\mathcal{N}$ such that $\mathcal{M} \tilde{v}_{i}=\tilde{w}_{i}$ and $\mathcal{N} \tilde{w}_{i}=\tilde{v}_{i}$ for each $i \in\{0, \ldots, n\}$. It follows that $\mathcal{M} \cdot \mathcal{N}$ and $\mathcal{N} \cdot \mathcal{M}$ are equal to the identity matrix. For each $i \in\{0, \ldots, 1\}$ the last coordinate of $\tilde{v}_{i}$, as well as the last coordinate of $\tilde{w}_{i}$, coincide with $\operatorname{den}\left(v_{i}\right)=\operatorname{den}\left(w_{i}\right)$. Thus $\mathcal{M}$ and $\mathcal{N}$ have the form

$$
\mathcal{M}=\left(\begin{array}{c|c}
\mathcal{U} & b \\
\hline 0, \ldots, 0 & 1
\end{array}\right) \quad \mathcal{N}=\left(\begin{array}{c|c}
\mathcal{V} & c \\
\hline 0, \ldots, 0 & 1
\end{array}\right)
$$

for some integer $(n \times n)$-matrices $\mathcal{U}$ and $\mathcal{V}$ and integer vectors $b, c \in \mathbb{Z}^{n}$. For each $i=0, \ldots, n$ the following holds: $\mathcal{M} \tilde{v}_{i}=\mathcal{M}\left(\operatorname{den}\left(v_{i}\right) \cdot\left(v_{i}, 1\right)\right)=\operatorname{den}\left(w_{i}\right) \cdot\left(\mathcal{U} v_{i}+b, 1\right)$. From $\mathcal{M} \tilde{v}_{i}=\tilde{w}_{i}=$ $\operatorname{den}\left(w_{i}\right) \cdot\left(w_{i}, 1\right)$ we obtain $\mathcal{U} v_{i}+b=w_{i}$. Similarly, $\mathcal{V} w_{i}+c=v_{i}$. In conclusion, both maps $\gamma(x)=\mathcal{U} x+b$ and $\mu(y)=\mathcal{V} y+c$ are in $\mathrm{GL}(n, \mathbb{Z}) \ltimes \mathbb{Z}^{n}$. Further, $\gamma^{-1}=\mu$ and $\gamma\left(v_{i}\right)=w_{i}$ for each $i \in\{0, \ldots, n\}$.

Lemma 2. Let $F \subseteq \mathbb{R}^{n}$ be an e-dimensional rational affine space $(e=0, \ldots, n)$, and $v_{0}$ a rational point lying in $F$. Then there exist rational points $v_{1}, \ldots, v_{e} \in F$ such that the simplex $\operatorname{conv}\left(v_{0}, \ldots, v_{e}\right)$ is regular.

Proof. The primitive integer vector $\tilde{v}_{0} \in \mathbb{R}^{n}$ is part of a base $\left(b_{0}=\tilde{v}_{0}, b_{1}, \ldots, b_{n}\right)$ of the free abelian group $\mathbb{Z}^{n+1}$. We may choose the integer vectors $b_{1}, \ldots, b_{e}$ in such a way that, for each $i=1, \ldots, e$, the uniquely determined rational point $v_{i}$ in $\mathbb{R}^{n}$ with $\tilde{v}_{i}=b_{i}$ belongs to $F$. It follows that $\operatorname{conv}\left(v_{0}, \ldots, v_{e}\right)$ is a regular $e$-simplex contained in $F$.

\section{Classification of Rational affine subspaces of $\mathbb{R}^{n}$}

Given two rational affine spaces $F, G \subseteq \mathbb{R}^{n}$, the main result of this section (Theorem 8 ) provides a necessary and sufficient condition for the existence of $\gamma \in \mathrm{GL}(n, \mathbb{Z}) \ltimes \mathbb{Z}^{n}$ such that $\gamma(F)=G$. This is a first step for our complete classification of $\left(\mathrm{GL}(n, \mathbb{Z}) \ltimes \mathbb{Z}^{n}\right)$-orbits in Section 4 . 
Definition 3. For every non-empty rational affine space $F \subseteq \mathbb{R}^{n}$ we let

$$
d_{F}=\min \left\{\operatorname{den}(v) \mid v \in F \cap \mathbb{Q}^{n}\right\} .
$$

We have the following useful characterization of $d_{F}$ :

Lemma 4. Let $F \subseteq \mathbb{R}^{n}$ be an e-dimensional rational affine space $(e=0, \ldots, n)$. For any regular $e$-simplex $\operatorname{conv}\left(v_{0}, \ldots, v_{e}\right) \subseteq F, \quad d_{F}=\operatorname{gcd}\left(\operatorname{den}\left(v_{0}\right), \ldots, \operatorname{den}\left(v_{e}\right)\right)$.

Proof. Let $d=\operatorname{gcd}\left(\operatorname{den}\left(v_{0}\right), \ldots, \operatorname{den}\left(v_{e}\right)\right)$. For each $v \in F \cap \mathbb{Q}^{n}, \tilde{v} \in \mathbb{Z} \tilde{v}_{0}+\cdots+\mathbb{Z} \tilde{v}_{e}$. Since $\operatorname{den}(v)$ is a linear combination of $\operatorname{den}\left(v_{0}\right), \ldots, \operatorname{den}\left(v_{e}\right)$ with integer coefficients, then $d \leq d_{F}$. Conversely, for suitable integers $m_{0}, \ldots, m_{e}$ we can write $d=m_{0} \cdot \operatorname{den}\left(v_{0}\right)+\cdots+m_{e} \cdot \operatorname{den}\left(v_{e}\right)$. The uniquely determined rational point $w \in F \cap \mathbb{Q}^{n}$ with $\tilde{w}=m_{0} \cdot \tilde{v}_{0}+\cdots+m_{e} \cdot \tilde{v}_{e} \operatorname{satisfies} \operatorname{den}(w)=d$. Thus $d_{F} \leq d$.

Lemma 5. Let $F \subseteq \mathbb{R}^{n}$ be an e-dimensional rational affine space $(e=0, \ldots, n)$. Then there are rational points $v_{0}, \ldots, v_{e}$ lying in $F$ such that $\operatorname{conv}\left(v_{0}, \ldots, v_{e}\right)$ is a regular e-simplex and $\operatorname{den}\left(v_{i}\right)=d_{F}$ for each $i \in\{0, \ldots, e\}$.

Proof. Pick $v_{0} \in F \cap \mathbb{Q}^{n}$ with $\operatorname{den}\left(v_{0}\right)=d_{F}$. Lemma 2 yields rational points $w_{1}, \ldots, w_{e} \in F$ such that $\operatorname{conv}\left(v_{0}, w_{1}, \ldots, w_{e}\right)$ is regular. If $\operatorname{den}\left(w_{i}\right)>d_{F}$ for some $i \in\{1, \ldots, e\}$, then let the integer $m_{i}$ be uniquely determined by writing $m_{i} \cdot d_{F}<\operatorname{den}\left(w_{i}\right) \leq\left(m_{i}+1\right) \cdot d_{F}$. Let $v_{i} \in F \cap \mathbb{Q}^{n}$ be the unique rational point with $\tilde{v}_{i}=\tilde{w}_{i}-m_{i} \tilde{v}_{0}$. Then $d_{F} \leq \operatorname{den}\left(v_{i}\right)=\operatorname{den}\left(w_{i}\right)-m_{i} \cdot \operatorname{den}\left(v_{0}\right)=$ $\operatorname{den}\left(w_{i}\right)-m_{i} \cdot d_{F} \leq d_{F}$. The new $(e+1)$-tuple of integer vectors $\left(\tilde{v}_{0}, \tilde{v}_{1}, \ldots, \tilde{v}_{e}\right)$ can be completed to a base of $\mathbb{Z}^{n+1}$, precisely as $\left(\tilde{v}_{0}, \tilde{w}_{1} \ldots, \tilde{w}_{e}\right)$ does. $\operatorname{Thus} \operatorname{conv}\left(v_{0}, \ldots, v_{e}\right)$ is regular.

Definition 6. Let $F \subseteq \mathbb{R}^{n}$ be an $e$-dimensional rational affine space. If $0 \leq e<n$ we define

$$
c_{F}=\min \left\{\operatorname{den}(v) \mid v \in \mathbb{Q}^{n} \backslash F \text { and } \exists v_{0}, \ldots, v_{e} \in F \cap \mathbb{Q}^{n} \text { with } \operatorname{conv}\left(v, v_{0}, \ldots, v_{e}\right) \text { regular }\right\} .
$$

If $e=n$ we define $c_{F}=1$.

Lemma 7. Let $F \subseteq \mathbb{R}^{n}$ be an e-dimensional rational affine space $(e=0, \ldots, n)$. Then there are rational points $v_{0}, \ldots, v_{n}$ such that

(i) $v_{0}, \ldots, v_{e} \in F$;

(ii) $\operatorname{den}\left(v_{i}\right)=d_{F}$ for all $i \in\{0, \ldots, e\}$;

(iii) $\operatorname{den}\left(v_{i}\right)=c_{F}$ for all $i \in\{0, \ldots, n\} \backslash\{0, \ldots, e\}$;

(iv) $\operatorname{conv}\left(v_{0}, \ldots, v_{n}\right)$ is a regular $n$-simplex (whence in particular, $v_{e+1}, \ldots, v_{n} \notin F$ ).

Proof. In case $e=n$, the result follows directly from Lemma 5. In case $e<n$, let $w_{0}, \ldots, w_{n} \in \mathbb{Q}^{n}$ be such that $w_{0}, \ldots, w_{e} \in F, \operatorname{conv}\left(w_{0}, \ldots, w_{n}\right)$ is regular, and $\operatorname{den}\left(w_{e+1}\right)=c_{F}$. Lemma 5 yields rational points $v_{0}, \ldots, v_{e} \in F$ such that $\operatorname{conv}\left(v_{0}, \ldots, v_{e}\right)$ is regular and $\operatorname{den}\left(v_{i}\right)=d_{F}$ for each $0 \leq i \leq e$. For every $v \in F \cap \mathbb{Q}^{n}$ we can write $\tilde{v} \in \mathbb{Z} \tilde{v}_{0}+\cdots+\mathbb{Z} \tilde{v}_{e}$, whence in particular $\mathbb{Z} \tilde{v}_{0}+\cdots+\mathbb{Z} \tilde{v}_{e}=\mathbb{Z} \tilde{w}_{0}+\cdots+\mathbb{Z} \tilde{w}_{e}$. Therefore, $\left\{\tilde{v}_{0}, \ldots, \tilde{v}_{e}, \tilde{w}_{e+1}, \ldots, \tilde{w}_{n}\right\}$ is a base of $\mathbb{Z}^{n+1}$, and the $n$-simplex $T_{0}=\operatorname{conv}\left(v_{0}, \ldots, v_{e}, w_{e+1}, \ldots, w_{n}\right)$ is regular. In order to obtain from $w_{e+1}, \ldots, w_{n}$ the points $v_{e+1}, \ldots, v_{n}$ satisfying (iii)-(iv), we preliminarily set $v_{e+1}=w_{e+1}$. Similarly, for each $i=e+2, \ldots, n$ such that $\operatorname{den}\left(w_{i}\right)=c_{F}$ we set $v_{i}=w_{i}$. If $c_{F}=\operatorname{den}\left(w_{e+2}\right)=\cdots=\operatorname{den}\left(w_{n}\right)$, we are done. Otherwise, for all $i \in\{e+2, \ldots, n\}$ with $\operatorname{den}\left(w_{i}\right)>c_{F}$, letting the integer $m_{i}$ be the unique solution of $m_{i} \cdot c_{F}<\operatorname{den}\left(w_{i}\right) \leq\left(m_{i}+1\right) \cdot c_{F}$, we let the rational point $v_{i} \in \mathbb{R}^{n}$ be uniquely determined by the stipulation $\tilde{v}_{i}=\tilde{w}_{i}-m_{i} \cdot \tilde{w}_{e+1}$. We then have $c_{F} \leq \operatorname{den}\left(v_{i}\right)=$ $\operatorname{den}\left(w_{i}\right)-m_{i} \cdot \operatorname{den}\left(w_{e+1}\right)=\operatorname{den}\left(w_{i}\right)-m_{i} \cdot c_{F} \leq c_{F}$. The $n$-simplex $T=\operatorname{conv}\left(v_{0}, \ldots, v_{n}\right)$ obtained from $T_{0}$ in this way is regular, and has the desired properties.

The following theorem yields the classification of $\left(\mathrm{GL}(n, \mathbb{Z}) \ltimes \mathbb{Z}^{n}\right)$-orbits of rational affine spaces: the complete classifier is just a triplet of positive integers. As usual, for every affine space $F \subseteq \mathbb{R}^{n}$, $\operatorname{dim}(F)$ denotes its dimension.

Theorem 8. Let $F, G \subseteq \mathbb{R}^{n}$ be non-empty rational affine spaces. Then following conditions are equivalent: 
(i) There exists $\gamma \in \mathrm{GL}(n, \mathbb{Z}) \ltimes \mathbb{Z}^{n}$ such that $\gamma(F)=G$;

(ii) $\left(\operatorname{dim}(F), d_{F}, c_{F}\right)=\left(\operatorname{dim}(G), d_{G}, c_{G}\right)$.

Proof. (i) $\Rightarrow$ (ii) Since $\gamma \in \mathrm{GL}(n, \mathbb{Z}) \ltimes \mathbb{Z}^{n}$ is a bijective affine transformation, $\operatorname{dim}(F)=\operatorname{dim}(\gamma(F))=$ $\operatorname{dim}(G)$. The identities $d_{F}=d_{G}$ and $c_{F}=c_{G}$ follow directly from Definitions 3 and 6 , because $\gamma$ preserves denominators (Lemma 1).

(ii) $\Rightarrow(\mathrm{i})$. Let $v_{0}, \ldots, v_{n} \in \mathbb{Q}^{n}$ satisfy conditions (i)-(iv) of Lemma 7 relative to $F$. Similarly let $w_{0}, \ldots, w_{n} \in \mathbb{Q}^{n}$ satisfy the same conditions relative to $G$. By hypothesis, $\operatorname{den}\left(v_{i}\right)=d_{F}=$ $d_{G}=\operatorname{den}\left(w_{i}\right)$ for each $0 \leq i \leq \operatorname{dim}(F)=\operatorname{dim}(G)$ and $\operatorname{den}\left(v_{i}\right)=c_{F}=c_{G}=\operatorname{den}\left(w_{i}\right)$ for each $i \in\{0, \ldots, n\} \backslash\{0, \ldots, \operatorname{dim}(F)\}$. Lemma 1 yields $\gamma \in \mathrm{GL}(n, \mathbb{Z}) \ltimes \mathbb{Z}^{n}$ such that $\gamma\left(v_{i}\right)=w_{i}$ for each $i \in\{0, \ldots, n\}$. Since $\gamma$ is an affine transformation and $F=\operatorname{aff}\left(v_{0}, \ldots, v_{e}\right)$, then $G=$ $\operatorname{aff}\left(w_{0}, \ldots, w_{e}\right)=\operatorname{aff}\left(\gamma\left(v_{0}\right), \ldots, \gamma\left(v_{e}\right)\right)=\gamma(F)$.

As shown by the next three results, in many cases the parameter $c_{F}$ has no role in the classification of $\left(\mathrm{GL}(n, \mathbb{Z}) \ltimes \mathbb{Z}^{n}\right)$-orbits of rational affine spaces.

Lemma 9. Let $F \subseteq \mathbb{R}^{n}$ be a rational affine space. If $0 \leq \operatorname{dim}(F)<n-1$ then $c_{F}=1$. If $\operatorname{dim}(F)=n-1$, then $\operatorname{gcd}\left(c_{F}, d_{F}\right)=1$ and $1 \leq c_{F} \leq \max \left(1, d_{F} / 2\right)$.

Proof. Choose points $v_{0}, \ldots, v_{n} \in \mathbb{R}^{n}$ satisfying conditions (i)-(iv) in Lemma 7. Since $\tilde{v}_{0}, \ldots, \tilde{v}_{n}$ is a base of $\mathbb{Z}^{n+1}$, some linear combination of $\operatorname{den}\left(v_{0}\right), \ldots, \operatorname{den}\left(v_{n}\right)$ with integer coefficients equals 1 . Thus $\operatorname{gcd}\left(d_{F}, c_{F}\right)=1$. A moment's reflection shows that $c_{F} \leq d_{F}$. So, in case $d_{F}=1$ then $c_{F}=1$, and the result follows. On the other hand, in case $d_{F}>1$, from $\operatorname{gcd}\left(d_{F}, c_{F}\right)=1$ and $c_{F} \leq d_{F}$, it follows that $c_{F}<d_{F}$.

Let $e=\operatorname{dim}(F)$.

If $e<n-1$, then $v_{e+1} \neq v_{n}$ and (by Lemma $7\left(\right.$ iii)) $\operatorname{den}\left(v_{e+1}\right)=\operatorname{den}\left(v_{n}\right)=c_{F}$. Let $v \in \mathbb{Q}^{n}$ be such that $\tilde{v}=\tilde{v}_{0}-\tilde{v}_{n}$. Then $\left\{\tilde{v}_{0}, \ldots, \tilde{v}_{n-1}, \tilde{v}\right\}$ is a base of $\mathbb{Z}^{n+1}$. By Definition 6 , $c_{F} \leq \operatorname{den}(v)=$ $d_{F}-c_{F}$. Let $m$ be the unique integer such that $m \cdot c_{F}<\operatorname{den}(v) \leq(m+1) \cdot c_{F}$. Also let the rational point $w \in \mathbb{R}^{n}$ be defined by $\tilde{w}=\tilde{v}-m \cdot \tilde{v}_{e+1}$. Observe that $\left\{\tilde{v}_{0}, \ldots, \tilde{v}_{n-1}, \tilde{w}\right\}$ is a base of $\mathbb{Z}^{n+1}$. The minimality property of $c_{F}$ entails $c_{F} \leq \operatorname{den}(w)=\operatorname{den}(v)-m \cdot \operatorname{den}\left(v_{e+1}\right)=\operatorname{den}(v)-m \cdot c_{F} \leq c_{F}$. Then $\operatorname{den}(v)=d_{F}-c_{F}=(m+1) \cdot c_{F}$, whence $d_{F}=(m+2) \cdot c_{F}$. Since $\operatorname{gcd}\left(d_{F}, c_{F}\right)=1$, then $c_{F}=1$.

If $e=n-1$, then $\operatorname{den}\left(v_{n}\right)=c_{F}$. Let $v \in \mathbb{Q}^{n}$ be such that $\tilde{v}=\tilde{v}_{0}-\tilde{v}_{n}$. Then $\left\{\tilde{v}_{0}, \ldots, \tilde{v}_{n-1}, \tilde{v}\right\}$ is a base of $\mathbb{Z}^{n+1}$. The minimality property of $c_{F}$ yields the inequality $c_{F} \leq \operatorname{den}(v)=d_{F}-c_{F}$, that is, $c_{F} \leq d_{F} / 2$.

Corollary 10. Let $F, G \subseteq \mathbb{R}^{n}$ be non-empty rational affine spaces. Suppose $\operatorname{dim}(F)=\operatorname{dim}(G) \neq$ $n-1$. Then there exists $\gamma \in \mathrm{GL}(n, \mathbb{Z}) \ltimes \mathbb{Z}^{n}$ such that $\gamma(F)=G$ iff $d_{F}=d_{G}$.

Corollary 11. Let $F, G \subseteq \mathbb{R}^{n}$ be non-empty rational affine spaces, with $d_{F}=d_{G} \in\{1,2,3,4,6\}$. Then there exists $\gamma \in \mathrm{GL}(n, \mathbb{Z}) \ltimes \mathbb{Z}^{n}$ such that $\gamma(F)=G$ iff $\operatorname{dim}(F)=\operatorname{dim}(G)$.

The following result will find repeated use in the sequel:

Proposition 12. Let $n \in\{1,2, \ldots\}$ and $e, d, c$ be integers such that

(i) $e \in\{0, \ldots, n-1\}, d \in\{1,2, \ldots\}$, and $c \in\{1, \ldots, \max (1, d / 2)\}$;

(ii) $\operatorname{gcd}(d, c)=1$;

(iii) if $e \neq n-1$, then $c=1$.

Then for some integer $p \in\{1, \ldots, d\}$ with $\operatorname{gcd}(p, d)=1$, the rational affine space

$$
F=\left\{\left(y_{1}, \ldots, y_{n}\right) \in \mathbb{R}^{n} \mid y_{e+1}=\cdots=y_{n}=\frac{p}{d}\right\}
$$

satisfies $\left(\operatorname{dim}(F), d_{F}, c_{F}\right)=(e, d, c)$.

Proof. By (ii), there exist $p \in\{1, \ldots, d\}$ and $q=0,1, \ldots$ such that

$$
p \cdot c-q \cdot d=1
$$


The rational affine space $F=\left\{\left(y_{1}, \ldots, y_{n}\right) \in \mathbb{R}^{n} \mid y_{e+1}=\cdots=y_{n}=\frac{p}{d}\right\}$ satisfies $\operatorname{dim}(F)=e$ and $\operatorname{gcd}(p, d)=1$. Let the rational points $v_{0}, \ldots, v_{e} \in \mathbb{R}^{n}$ be defined as follows, for each $i \in\{0, \ldots, e\}$ and $j \in\{1, \ldots, n\}$ :

$$
\left(v_{i}\right)_{j}=j \text { th coordinate of } v_{i}= \begin{cases}\frac{p}{d} & \text { if } j \in\{e+1, \ldots, n\} \\ 0 & \text { if } j \in\{1, \ldots, e\} \backslash\{i\} \\ \frac{1}{d} & \text { if } j=i\end{cases}
$$

Thus in particular, $v_{0}=(\underbrace{0, \ldots, 0}_{e \text { times }}, \underbrace{p / d, \ldots, p / d}_{n-e \text { times }})$. By definition, $\operatorname{den}\left(v_{0}\right)=\cdots=\operatorname{den}\left(v_{e}\right)=d$.

To prove the identities $d_{G}=d$ and $c_{G}=c$ we argue by cases.

Case 1: $c=1$. We then set $p=1$ and $q=0$. Let $\xi_{1}, \ldots, \xi_{n}$ denote the canonical base of the vector space $\mathbb{R}^{n}$, and $\mathbf{0}=(0, \ldots, 0)$ the origin of $\mathbb{R}^{n}$. Then the $n$-simplex $\operatorname{conv}\left(v_{0}, \ldots, v_{e}, \mathbf{0}, \xi_{e+2}, \ldots, \xi_{n}\right)$ is regular. As a matter of fact, the integer $(n+1) \times(n+1)$-matrix $\mathcal{T}$ whose rows are the vectors $\tilde{v}_{1}, \ldots, \tilde{v}_{e}, \tilde{v}_{0}, \tilde{\xi}_{e+2}, \ldots, \tilde{\xi}_{n}, \tilde{\mathbf{0}}$, is lower triangular with all diagonal elements $=1$. By Lemma 4, $d_{F}=\operatorname{gcd}\left(\operatorname{den}\left(v_{0}\right), \ldots, \operatorname{den}\left(v_{e}\right)\right)=d$. By Definition $6, c_{F}=1=c$.

Case 2: $c \neq 1$. By (iii), $e=n-1$. Let the point $v \in \mathbb{R}^{n}$ be defined by $v=(0, \ldots, 0, q / c)$. Then $\operatorname{den}(v)=c$. Since $p \cdot c-q \cdot d=1$, the $n$-simplex $\operatorname{conv}\left(v_{0}, \ldots, v_{n-1}, v\right)$ is regular. By Lemma 4 , $d_{F}=\operatorname{gcd}\left(\operatorname{den}\left(v_{0}\right), \ldots, \operatorname{den}\left(v_{n-1}\right)\right)=d$. By Definition $6, c_{F} \leq c$. There is $w \in \mathbb{Q}^{n}$ such that $\operatorname{den}(w)=c_{F}$ and $\operatorname{conv}\left(v_{0}, \ldots, v_{n-1}, w\right)$ is a regular $n$-simplex. Since $\left\{\tilde{v}_{1}, \ldots, \tilde{v}_{n}, \tilde{w}\right\}$ is a basis of $\mathbb{Z}^{n+1}, \quad \tilde{v}$ is a linear combination of $\left\{\tilde{v}_{1}, \ldots, \tilde{v}_{n}, \tilde{w}\right\}$ with integer coefficients. Since $p \cdot c-q \cdot d=1$, we easily see that $c=c_{F}+k \cdot d$ for some $k \in \mathbb{Z}$. Using now (i) and Lemma 9 we obtain $c_{F}=c$, which completes the proof.

\section{4. $\left(\mathrm{GL}(n, \mathbb{Z}) \ltimes \mathbb{Z}^{n}\right)$-ORBIT CLASSIFICATION}

For each $x=\left(x_{1}, \ldots, x_{n}\right) \in \mathbb{R}^{n}$ let

$$
F_{x}=\bigcap\left\{F \subseteq \mathbb{R}^{n} \mid x \in F \text { and } F \text { is a rational affine space }\right\} .
$$

Also let the group $G_{x}$ be defined by

$$
G_{x}=\mathbb{Z}+x_{1} \mathbb{Z}+\cdots+x_{n} \mathbb{Z}
$$

In other words, $G_{x}$ is the subgroup of the additive group $\mathbb{R}$ generated by 1 together with the coordinates of $x$.

Lemma 13. For each $x \in \mathbb{R}^{n}$ and $\gamma \in \mathrm{GL}(n, \mathbb{Z}) \ltimes \mathbb{Z}^{n}, \gamma\left(F_{x}\right)=F_{\gamma(x)}$ and $G_{x}=G_{\gamma(x)}$.

Proof. Let $y=\gamma(x)$. Since $\gamma$ and $\gamma^{-1}$ preserve rational affine spaces we have the inclusions $F_{y} \subseteq \gamma\left(F_{x}\right)$ and $F_{x} \subseteq \gamma^{-1}\left(F_{y}\right)$, whence $F_{y} \subseteq \gamma\left(F_{x}\right) \subseteq \gamma\left(\gamma^{-1}\left(F_{y}\right)\right)=F_{y}$.

Let $x=\left(x_{1}, \ldots, x_{n}\right)$ and $y=\left(y_{1}, \ldots, y_{n}\right)$. Let $\mathcal{U} \in \mathbb{Z}^{n \times n}$ and $b \in \mathbb{Z}^{n}$ be such that $\gamma(z)=\mathcal{U} z+b$ for each $z \in \mathbb{R}^{n}$. Since all terms of $\mathcal{U}$ and all coordinates of $b$ are integers, then $y_{i} \in G_{x}$ for each $i \in\{1, \ldots, n\}$. Therefore, $G_{y} \subseteq G_{x}$. The converse inclusion follows replacing $\gamma$ by $\gamma^{-1}$.

Lemma 14. For each $x=\left(x_{1}, \ldots, x_{n}\right) \in \mathbb{R}^{n}, \quad \operatorname{rank}\left(G_{x}\right)=\operatorname{dim}\left(F_{x}\right)+1$ and $d_{F_{x}}=\max \{k \in \mathbb{Z} \mid$ $\left.1 / k \in G_{x}\right\}$.

Proof. Let $e=\operatorname{dim}\left(F_{x}\right)$ and $d=d_{F_{x}}$. We argue by cases.

Case 1: $e=n$. Then $F_{x}=\mathbb{R}^{n}$. Suppose the integers $k_{0}, \ldots, k_{n}$ satisfy $k_{0}+k_{1} \cdot x_{1}+\cdots+k_{n} \cdot x_{n}=0$. Letting the vector $v \in \mathbb{Z}^{n}$ be defined by $v=\left(k_{1}, \ldots, k_{n}\right)$, we have the identity $\langle x, v\rangle=-k_{0}$. Since $F_{x}=\mathbb{R}^{n}$ then necessarily $k_{1}=\cdots=k_{n}=0$, and hence $k_{0}=\langle x, v\rangle=0$. We have just proved that $\operatorname{rank}\left(G_{x}\right)=n+1$, and $G_{x} \cap \mathbb{Q}=\mathbb{Z}$. It follows that $\max \left\{k \in \mathbb{Z} \mid 1 / k \in G_{x}\right\}=1$. By Definition 3, $d_{F_{x}}=1$, which settles the proof of this case. 
Case 2: $e<n$. Then Proposition 12 and Theorem 8 jointly yield $p \in\{1, \ldots, d\}$ and $\gamma \in \mathrm{GL}(n, \mathbb{Z}) \ltimes$ $\mathbb{Z}^{n}$ such that $\operatorname{gcd}(p, d)=1$ and

$$
\gamma\left(F_{x}\right)=\left\{\left(y_{1}, \ldots, y_{n}\right) \in \mathbb{R}^{n} \mid y_{i}=\frac{p}{d} \text { for each } i=e+1, \ldots, n\right\}=F .
$$

Let $\gamma(x)=y=\left(y_{1}, \ldots, y_{e}, \frac{p}{d}, \ldots, \frac{p}{d}\right)$. By Lemma 13, $G_{y}=G_{x}$ and $F_{y}=\gamma\left(F_{x}\right)=F$. Let $k_{0}, \ldots, k_{e}$ be such that $k_{0} \cdot \frac{1}{d}+k_{1} \cdot y_{1}+\cdots+k_{e} \cdot y_{e}=0$. Then the rational point $v=\left(k_{1}, \ldots, k_{e}, 0, \ldots, 0\right)$ satisfies $\langle v, y\rangle \in \mathbb{Q}$. Since $F_{y}=F$, we also have $\langle v, z\rangle \in \mathbb{Q}$ for each $z \in F$, whence $v=0$ and $k_{0} \cdot \frac{1}{d}+h_{1} \cdot y_{1}+\cdots+k_{e} \cdot y_{e}=k_{0} \cdot \frac{1}{d}=0$. Having thus proved that $k_{i}=0$ for each $i \in\{0, \ldots, e\}$, it follows that $\operatorname{rank}\left(G_{x}\right)=\operatorname{rank}\left(G_{y}\right)=e+1$.

Assume the integer $k>0$ satisfies $\frac{1}{k} \in G_{x}=G_{y}$. Then there exist integers $k_{0}, \ldots, k_{e}$ satisfying $\frac{1}{k}=k_{0} \cdot \frac{1}{d}+k_{1} \cdot y_{1}+\cdots+k_{e} \cdot y_{e}$. It follows that $1=k \cdot k_{0} \cdot \frac{1}{d}+k \cdot k_{1} \cdot y_{1}+\cdots+k \cdot k_{e} \cdot y_{e}$. From $\operatorname{rank}\left(G_{y}\right)=e+1$ it follows that $k \cdot k_{0}=d$. Thus $k \leq d$, and $d=\max \left\{k \in \mathbb{Z} \mid \frac{1}{k} \in G_{x}\right\}$. This settles Case 2 and completes the proof.

We are now ready to prove the main result of this paper and its corollaries, thus providing a complete classification of $\left(\mathrm{GL}(n, \mathbb{Z}) \ltimes \mathbb{Z}^{n}\right)$-orbits.

Theorem 15. For all $x, y \in \mathbb{R}^{n}$ the following conditions are equivalent:

(i) there exists $\gamma \in \mathrm{GL}(n, \mathbb{Z}) \ltimes \mathbb{Z}^{n}$ such that $\gamma(x)=y$;

(ii) $\left(G_{x}, c_{F_{x}}\right)=\left(G_{y}, c_{F_{y}}\right)$.

Proof. (i) $\Rightarrow$ (ii) follows directly from Theorem 8 and Lemma 13. (ii) $\Rightarrow$ (i) We argue by cases:

Case 1: $\operatorname{rank}\left(G_{x}\right)=n+1$. By Lemma 14, $\operatorname{dim}\left(F_{x}\right)=\operatorname{rank}\left(G_{x}\right)-1=\operatorname{rank}\left(G_{y}\right)-1=\operatorname{dim}\left(F_{y}\right)=n$. Since $G_{x}=G_{y}$, there are $(n+1) \times(n+1)$ integer matrices $\mathcal{M}$ and $\mathcal{N}$ such that $\mathcal{M}\left(x_{1}, \ldots, x_{n}, 1\right)=$ $\left(y_{1}, \ldots, y_{n}, 1\right)$ and $\mathcal{N}\left(y_{1}, \ldots, y_{n}, 1\right)=\left(x_{1}, \ldots, x_{n}, 1\right)$. Thus $\mathcal{M}$ and $\mathcal{N}$ have the form

$$
\mathcal{M}=\left(\begin{array}{c|c}
\mathcal{U} & b \\
\hline 0, \ldots, 0 & 1
\end{array}\right) \quad \mathcal{N}=\left(\begin{array}{c|c}
\mathcal{V} & c \\
\hline 0, \ldots, 0 & 1
\end{array}\right)
$$

and $\mathcal{N} \mathcal{M}\left(x_{1}, \ldots, x_{n}, 1\right)=\left(x_{1}, \ldots, x_{n}, 1\right)$. Since $\operatorname{rank}\left(G_{x}\right)=n+1, \mathcal{N} \mathcal{M}$ is the identity $(n+1) \times$ $(n+1)$-matrix and $\mathcal{N}=\mathcal{M}^{-1}$. Thus the affine map $\gamma: \mathbb{R}^{n} \rightarrow \mathbb{R}^{n}$ defined by $\gamma(z)=\mathcal{U} z+b$ belongs to $\mathrm{GL}(n, \mathbb{Z}) \ltimes \mathbb{Z}^{n}$ and $\gamma(x)=y$.

Case 2: $1 \leq \operatorname{rank}\left(G_{x}\right) \leq n$. By Lemma 14 $\operatorname{dim}\left(F_{x}\right)=\operatorname{rank}\left(G_{x}\right)-1=\operatorname{rank}\left(G_{y}\right)-1=\operatorname{dim}\left(F_{y}\right)$, and $d_{F_{x}}=\max \left\{k \in \mathbb{Z} \mid 1 / k \in G_{x}\right\}=\max \left\{k \in \mathbb{Z} \mid 1 / k \in G_{y}\right\}=d_{F_{y}}$. Let us use the abbreviations

$$
e=\operatorname{dim}\left(F_{x}\right)=\operatorname{dim}\left(F_{y}\right), \quad d=d_{F_{x}}=d_{F_{y}}, c=c_{F_{x}}=c_{F_{y}} .
$$

Proposition 12 yields an integer $p$ with $1 \leq p \leq d$ such that the affine space

$$
F=\left\{\left(y_{1}, \ldots, y_{n}\right) \in \mathbb{R}^{n} \mid y_{i}=\frac{p}{d} \text { for each } i \in\{e+1, \ldots, n\}\right\}
$$

satisfies $\left(\operatorname{dim}(F), d_{F}, c_{F}\right)=(e, d, c)$. By Theorem 8 there are $\gamma_{1}, \gamma_{2} \in \mathrm{GL}(n, \mathbb{Z}) \ltimes \mathbb{Z}^{n}$ such that $\gamma_{1}\left(F_{x}\right)=\gamma_{2}\left(F_{y}\right)=F$.

Writing for short $x^{\prime}=\gamma_{1}(x), y^{\prime}=\gamma_{2}(y)$, the proof of (i) amounts to showing

$$
y^{\prime} \in \operatorname{orb}\left(x^{\prime}\right) \text {. }
$$

To this purpose, we define the rational points $v_{0}, \ldots, v_{e} \in F$ by stipulating that for all $i \in\{0, \ldots, e\}$ and $j \in\{1, \ldots, n\}$ :

$$
\left(v_{i}\right)_{j}=j \text { th coordinate of } v_{i}= \begin{cases}\frac{p}{d} & \text { if } j \in\{e+1, \ldots, n\} \\ 0 & \text { if } j \in\{1, \ldots, e\} \backslash\{i\} \\ \frac{1}{d} & \text { if } j=i\end{cases}
$$

Then $F$ coincides with aff $\left(v_{0}, \ldots, v_{e}\right)$, and $\operatorname{conv}\left(v_{0}, \ldots, v_{e}\right)$ is a regular $e$-simplex. Next let us define the map $\eta: F \rightarrow \mathbb{R}^{e}$ by

$$
\eta\left(z_{1}, \ldots, z_{n}\right)=\left(d \cdot z_{1}, \ldots, d \cdot z_{e}\right) \text { for all } z=\left(z_{1}, \ldots, z_{n}\right) \in F .
$$


Equivalently, letting $\xi_{1}, \ldots, \xi_{e} \in \mathbb{R}^{e}$ denote the vectors of the canonical base of $\mathbb{R}^{e}, \quad \eta$ is the unique affine transformation such that $\eta\left(v_{0}\right)=\mathbf{0}=$ the origin of $\mathbb{R}^{e}$, and $\eta\left(v_{i}\right)=\xi_{i}$ for each $i \in\{1, \ldots, e\}$. For any integer $m$ and subgroup $G$ of the additive group $\mathbb{R}$, let us use the self-explanatory notation $m \cdot G=\{m \cdot g \mid g \in G\}$. Then for every $z=\left(z_{1}, \ldots, z_{n}\right) \in F$ we can write

$$
G_{\eta(z)}=d \cdot z_{1} \mathbb{Z}+\cdots+d \cdot z_{e} \mathbb{Z}+\mathbb{Z}=d \cdot\left(z_{1} \mathbb{Z}+\cdots+z_{e} \mathbb{Z}+\frac{1}{d} \mathbb{Z}\right)=d \cdot G_{z} .
$$

In particular, $G_{\eta\left(x^{\prime}\right)}=d \cdot G_{x^{\prime}}=d \cdot G_{x}=d \cdot G_{y}=d \cdot G_{y^{\prime}}=G_{\eta\left(y^{\prime}\right)}$. Since $\operatorname{rank}\left(G_{\eta\left(x^{\prime}\right)}\right)=e+1=$ $\operatorname{rank}\left(G_{\eta\left(y^{\prime}\right)}\right)$, from Lemma 14 we obtain $\operatorname{dim}\left(F_{\eta\left(x^{\prime}\right)}\right)=e$. The proof of Case 1 above (with $e$ in place of $n)$ yields $\gamma_{3} \in \mathrm{GL}(e, \mathbb{Z}) \ltimes \mathbb{Z}^{e}$ such that

$$
\gamma_{3}\left(\eta\left(x^{\prime}\right)\right)=\eta\left(y^{\prime}\right)
$$

Since $\eta$ is one-to-one onto $\mathbb{R}^{e}$, there exist uniquely determined rational points $w_{0}, \ldots, w_{e} \in F$ such that

$$
\eta\left(w_{0}\right)=\gamma_{3}(\mathbf{0}) \text { and } \eta\left(w_{i}\right)=\gamma_{3}\left(\xi_{i}\right) \text { for each } i \in\{1, \ldots, e\} .
$$

By (2),

$$
\operatorname{den}\left(w_{i}\right)=d \cdot \operatorname{den}\left(\gamma_{3}\left(\xi_{i}\right)\right)=d, \text { for each } i \in\{1, \ldots, e\}
$$

Claim: The $e$-simplex $\operatorname{conv}\left(w_{0}, \ldots, w_{e}\right) \subseteq F$ is regular.

Indeed, for each $i \in\{1, \ldots, e\}$ we have $\left.\left.\widetilde{\eta\left(w_{i}\right.}\right)=\widetilde{\gamma_{3}\left(\xi_{i}\right.}\right)=\left(\gamma_{3}\left(\xi_{i}\right), 1\right)=\left(\eta\left(w_{i}\right), 1\right)$. Thus

$$
\left.\left\{\widetilde{\eta\left(w_{0}\right.}\right), \ldots, \widetilde{\eta\left(w_{e}\right)}\right\}=\left\{\left(\eta\left(w_{0}\right), 1\right), \ldots,\left(\eta\left(w_{e}\right), 1\right)\right\} \text { is a base of } \mathbb{Z}^{e} .
$$

In case $c=1$, the proof of Proposition 12 shows that $p=1$, whence

$$
\tilde{w}_{i}=d \cdot\left(w_{i}, 1\right)=\left(d \cdot w_{i}, d\right)=\left(\eta\left(w_{i}\right), 1, \ldots, 1, d\right) \text {, for each } i \in\{1, \ldots, e\} .
$$

Let $\mu_{1}, \ldots, \mu_{n+1}$ denote the canonical base of the free abelian group $\mathbb{Z}^{n+1}$. From (66)-(7) it follows that $\left\{\tilde{w}_{0}, \ldots, \tilde{w}_{e}, \mu_{e+2}, \ldots, \mu_{n+1}\right\}$ is a base of $\mathbb{Z}^{n+1}$. Thus the $e-\operatorname{simplex} \operatorname{conv}\left(w_{0}, \ldots, w_{e}\right)$ is regular.

In case $c \neq 1$, by Lemma 9 , $e=n-1$. Further, $\tilde{w}_{i}=d \cdot\left(w_{i}, 1\right)=\left(d \cdot w_{i}, d\right)=\left(\eta\left(w_{i}\right), p, d\right)$ for each $i \in\{0, \ldots, e\}$. The proof of Proposition 12 provides an integer $q \geq 1$ such that $p \cdot c-q \cdot d=1$. Upon defining $v=\left(0, \ldots, 0, \frac{q}{c}\right)$, from (6) it follows that the set of integer vectors $\left\{\left(\eta\left(w_{0}\right), p, d\right), \ldots,\left(\eta\left(w_{e}\right), p, d\right),(0, \ldots, 0, q, c)\right\}=\left\{\tilde{w}_{0}, \ldots, \tilde{w}_{e}, \tilde{v}\right\}$ is a base of $\mathbb{Z}^{n+1}$. Thus, $\operatorname{conv}\left(w_{0}, \ldots, w_{e}\right)$ is regular.

Our claim is settled.

As an immediate consequence of the regularity of the $e$-simplex $\operatorname{conv}\left(w_{0}, \ldots, w_{e}\right)$, also the $n$-simplex $\operatorname{conv}\left(w_{0}, \ldots, w_{e}, v_{e+1}, \ldots, v_{n}\right)$ is regular. By (5), $\operatorname{den}\left(w_{i}\right)=\operatorname{den}\left(v_{i}\right)=d$ for each $i \in\{0, \ldots, e\}$. Lemma 1 yields $\gamma_{4} \in \mathrm{GL}(n, \mathbb{Z}) \ltimes \mathbb{Z}^{n}$ such that $\gamma_{4}\left(v_{i}\right)=w_{i}$ for $i \in\{0, \ldots, e\}$, and $\gamma_{4}\left(v_{i}\right)=v_{i}$ for $i \in\{e+1, \ldots, n\}$. It follows that $\gamma_{4}(F)=F$.

Further,

$$
\eta\left(\gamma_{4}(z)\right)=\gamma_{3}(\eta(z)) \text { for each } z \in F .
$$

As a matter of fact, by (4) we can write $\eta\left(\gamma_{4}\left(v_{0}\right)\right)=\eta\left(w_{0}\right)=\gamma_{3}(\mathbf{0})=\gamma_{3}\left(\eta\left(v_{0}\right)\right)$ and $\eta\left(\gamma_{4}\left(v_{i}\right)\right)=$ $\eta\left(w_{i}\right)=\gamma_{3}\left(\xi_{i}\right)=\gamma_{3}\left(\eta\left(v_{i}\right)\right)$ for each $i=1, \ldots, n$. Then (8) follows, because $\eta, \gamma_{3}$ and $\gamma_{4}$ are affine transformations and $F=\operatorname{aff}\left(v_{0}, \ldots, v_{e}\right)$.

Since $\gamma_{4}\left(x^{\prime}\right) \in F$, from (3) and (8) we can write $\eta\left(\gamma_{4}\left(x^{\prime}\right)\right)=\gamma_{3}\left(\eta\left(x^{\prime}\right)\right)=\eta\left(y^{\prime}\right)$. Since the map $\eta: F \rightarrow \mathbb{R}^{e}$ is one-to-one, we finally obtain $\gamma_{4}\left(x^{\prime}\right)=y^{\prime}$, which proves (11) and completes the proof of the theorem.

Using Lemmas 9 and 14 we obtain the following special cases of Theorem 15.

Corollary 16. For all $x, y \in \mathbb{R}^{n}$, if $\operatorname{rank}\left(G_{x}\right) \neq n$, the following conditions are equivalent:

(i) There exists $\gamma \in \mathrm{GL}(n, \mathbb{Z}) \ltimes \mathbb{Z}^{n}$, such that $\gamma(x)=y$;

(ii) $G_{x}=G_{y}$. 
Corollary 17. For all $x, y \in \mathbb{R}^{n}$, if $\max \left\{k \in \mathbb{Z} \mid 1 / k \in G_{x}\right\} \in\{1,2,3,4,6\}$, the following conditions are equivalent:

(i) There exists $\gamma \in \mathrm{GL}(n, \mathbb{Z}) \ltimes \mathbb{Z}^{n}$, such that $\gamma(x)=y$;

(ii) $G_{x}=G_{y}$.

In conclusion, from Lemmas 9 and 14. Proposition 12 and Theorem 15 we obtain:

Corollary 18. The map $\operatorname{orb}(x) \mapsto\left(G_{x}, c_{F_{x}}\right)$ is a one-one correspondence between $\left(\mathrm{GL}(n, \mathbb{Z}) \ltimes \mathbb{Z}^{n}\right)$ orbits and pairs $(G, c)$, where $G$ is an arbitrary subgroup of the additive group $\mathbb{R}$ having rank $\leq n+1$ and containing 1 among its elements, and $c$ is an integer satisfying the following conditions: if $\operatorname{rank}(G) \neq n$ then $c=1$; if $\operatorname{rank}(G)=n$, letting $1 / d$ be the smallest positive rational in $G$, then $1 \leq c \leq \max (1, d / 2)$ and $\operatorname{gcd}(c, d)=1$. There are precisely $\max (1, \phi(d) / 2)$ possible values for $c$.

\section{REFERENCES}

[1] J.S. Dani, Density properties of orbits under discrete groups, J. Indian Math. Soc. 39 (1975) 189-218.

[2] A. Guilloux, A brief remark on orbits of $\operatorname{SL}(2, \mathbb{Z})$ in the Euclidean plane, Ergodic Theory Dynam. Systems 30 (2010) 1101-1109.

[3] G. Ewald, Combinatorial convexity and algebraic geometry, Grad. Texts in Math., Vol. 168, Springer-Verlag, New York, 1996.

[4] M. Laurent, A. Nogueira, Approximation to points in the plane by $\operatorname{SL}(2, \mathbb{Z})$-orbits, J. London Math. Soc. (2) 85 (2012) 409-429.

[5] D. Mundici, The Haar theorem for lattice-ordered abelian groups with order-unit, Discrete Contin. Dyn. Syst. 21 (2008) 537-549.

[6] D. Mundici, Invariant measure under the affine group over $\mathbb{Z}$, Combin. Probab. Comput. 23 (2014) 248-268.

[7] D. Mundici, Classifying $\left(\mathrm{GL}(2, \mathbb{Z}) \ltimes \mathbb{Z}^{2}\right)$-orbits by subgroups of $\mathbb{R}$, (preprint available at arXiv:1401.3708)

[8] A. Nogueira, Orbit distribution on $\mathbb{R}^{2}$ under the natural action of $\mathrm{SL}(2, \mathbb{Z})$, Indag. Math. (N.S.), 13 (2002) $103-124$

[9] A. Nogueira, Lattice orbit distribution on $\mathbb{R}^{2}$, Ergodic Theory Dynam. Systems 30 (2010) 1201-1214. Erratum, ibid., p. 1215.

[10] E. Witten, $\mathrm{SL}(2, \mathbb{Z})$ action on three-dimensional conformal field theories with abelian symmetry, In: From Fields to Strings: Circumnavigating Theoretical Physics, Ian Kogan Memorial Collection (in 3 volumes), M. Shifman et al., Eds., World Scientific, Singapore, (2005), pp. 1173-1200.

(L.M. Cabrer) Department of Statistics, Computer Science and Applications, "Giuseppe Parenti", University of Florence, Viale Morgagni 59 - 50134, Florence, Italy

E-mail address: 1.cabrer@disia.unifi.it

(D. Mundici) Department of Mathematics and Computer Science "Ulisse Dini", University of FloRence, Viale Morgagni 67/A, I-50134 Florence, Italy

E-mail address: mundici@math.unifi.it 